\title{
Genderstern und Binnen-I: Es ist Zeit, die Realität zu akzeptieren
}

\author{
Zu Rudolf Stöbers Beitrag ,Genderstern und Binnen-I. \\ $\mathrm{Zu}$ falscher Symbolpolitik in Zeiten eines zunehmenden \\ Illiberalismus“" (Publizistik, Heft 1, 2021)
}

\section{Thomas Hanitzsch}

Angenommen: 27. Februar 2021 / Online publiziert: 19. März 2021

(C) Der/die Autor(en) 2021

In der Rubrik Meinungsforum publizieren wir Meinungsbeiträge von Kommunikationswissenschaftler*innen, die ein für unser Fach relevantes und aktuelles Thema aufgreifen, das mitunter auch in der breiteren Öffentlichkeit kontrovers diskutiert wird. Einreichungen, die Perspektiven der Fachentwicklung und -politik zur Diskussion stellen, sind ebenfalls willkommen. Beiträge im Meinungsforum beruhen auf kommunikationswissenschaftlichen Befunden und Konzepten; sie werden redaktionell geprüft und redigiert, durchlaufen aber kein Double-Blind Peer Review. Sie stellen nicht notwendigerweise die Meinung von Herausgeberteam und Redaktion dar und sollen vor allem der Diskussion im Fach dienen. Um Debatten im Meinungsforum der Publizistik anzuregen, drucken wir gleichzeitig mindestens eine Replik ab; weitere Beiträge können in den nachfolgenden Heften erscheinen.

Zu dem Beitrag „,Genderstern und Binnen-I. Zu falscher Symbolpolitik in Zeiten eines zunehmenden Illiberalismus “ in der vorigen Ausgabe unserer Zeitschrift haben uns bis Redaktionsschluss sechs Repliken erreicht. Wir drucken auf den folgenden Seiten alle sechs Beiträge ungekürzt und alphabetisch nach den Namen der Autor*innen geordnet $a b$.

Die Red.

Rudolf Stöber hat sich an dieser Stelle in einem Meinungsbeitrag mit dem Thema Genderstern und Binnen-I und deren Verwendung in akademischer Prosa kritisch-polemisch auseinandergesetzt. Der Beitrag wiederholt dabei bekannte Positionen, ohne wirklich neue Argumente einzubringen, und stellt Gegenpositionen in ein falsches Licht. Angesichts des überwältigenden Forschungsstands zum Thema wirkt der Text sonderbar aus der Zeit gefallen. Zudem konnte der Verfasser offenbar nicht der Versuchung widerstehen, Verwender*innen von Genderstern und Binnen-I in die Nähe des Nationalsozialismus und SED-Regimes zu rücken.

Prof. Dr. T. Hanitzsch $(\bowtie)$

Institut für Kommunikationswissenschaft und Medienforschung, Ludwig-Maximilians-Universität München, Oettingenstr. 67, 80538 München, Deutschland

E-Mail: thomas.hanitzsch@ifkw.lmu.de 
Dabei wendet sich Rudolf Stöber zugegebenermaßen nicht gegen geschlechtergerechte Sprache per se. Ihm geht es insbesondere um die Verwendung von Genderstern und Binnen-I und die damit einhergehende „Beschädigung“ und „Verarmung“ der deutschen Sprache. Genderstern und Binnen-I sind „derzeit sprachnormwidrig“, schreibt Stöber. Ihre Verwendung gelte gemäß dem Rat für deutsche Rechtschreibung ,noch immer als Abweichung von der Norm“. Die deutschen Universitäten und ihre Vertreter*innen, die zur Einhaltung dieser Norm verpflichtet seien, würden zunehmend dagegen verstoßen. Fast wird unterstellt, dass die Universitäten mit ihren Bemühungen um inklusive, geschlechtergerechte Formulierungen dieser Couleur quasi Rechts-, wenigstens aber einen Tabubruch begingen. In der Konsequenz werde eine ,unglaubliche sprachliche Verarmung“ betrieben und „die Institution Sprache beschädigt“. Leser*innen mögen sich fragen, worin genau denn die Verarmung und der Schaden eigentlich bestehen. Vielfalt mit Verarmung gleichzusetzen scheint nicht nur lexikalisch abseitig. Was genau ist also das Problem? Ein ästhetisches Unwohlsein allein ist es nicht, schreibt Stöber selbst. Der Grund liegt tiefer. Aber dazu kommen wir später.

In Stöbers Meinungsbeitrag wird die deutsche Rechtschreibung als sakrosankt markiert und vordergründig gegen Versuche der sprachlichen Inklusion ins Feld geführt. Das Ziel ist die Verteidigung des linguistischen Status quo - eine häufige Argumentationsfigur von Gegner*innen von Genderstern und Binnen-I (und ihren verwandten Formen, vgl. Vergoossen et al. 2020). Die Rechtschreibung ist jedoch keine prinzipiell unveränderliche Konvention (man sehe sich nur die diversen Rechtschreibreformen der letzten Jahre an), die uns äußerlich vorgegeben ist. Sprache ist menschengemacht, und so ist es auch der Umgang mit ihr. Wir entscheiden, in welcher Form wir mit Sprache die gesellschaftlichen Wirklichkeiten repräsentieren und damit - ja, und auch das ist unbestritten - die Realität formen.

Wann haben wir als aufgeklärte Wissenschaft eigentlich beschlossen, dass eine prinzipiell formbare Konvention wie die deutsche Rechtschreibung einer unbestritten notwendigen Transformation gesellschaftlicher Realitäten im Wege stehen soll? Um es ganz klar zu sagen: Mit der Verwendung von Genderstern und Binnen-I wird unsere Welt nicht untergehen. Alles, was es braucht, ist die Bereitschaft zu etwas geistiger Flexibilität. Eine spannende Aufarbeitung der neuesten Erkenntnisse zur Flexibilität von Sprache im Allgemeinen und gendergerechter Sprache im Besonderen findet sich übrigens in einem lesenswerten Beitrag von Stefan Hartmann (2021) im aktuellen Leserforum der Zeitschrift Forschung \& Lehre.

Interessanterweise streitet Stöber in seinem Beitrag den Zusammenhang zwischen Sprache, Denken und Handeln nicht ab. So sei hier nur kurz darauf verwiesen, dass generisch maskuline Personenbezeichnungen stärker mit Männern assoziiert werden (vgl. Irmen und Linner 2005) und dass geschlechtergerechte Sprache zu einem stärkeren gedanklichen Einbezug von Frauen führt (vgl. z. B. Blake et al. 2008; Sczesny und Stahlberg 2001, 2005). Stöber merkt daher zutreffend an: „Sprache beeinflusst unser Denken [...] unreflektiert, nicht intentional.“

Dennoch missdeutet Stöber die Verwendung geschlechtergerechter Sprache schlicht als Markierung von Differenz. Denn damit würde eine „Trivialität“ angezeigt, ,,dass Menschen verschieden sind“. Aber hier irrt Stöber. Es geht den Verwender*innen von Genderstern und Binnen-I eben nicht um triviale Unterschiedlichkeit, 
sondern um geschlechterbezogene Ungleichheiten hinsichtlich der gesellschaftlichen Chancenverteilung und ihrer sozialen Konsequenzen. Geschlechtergerechte Sprache sollte daher angesehen werden als das, was sie ist: eine rhetorische Form der Inklusion.

Die Verwendung von Genderstern und Binnen-I sei noch immer eine Abweichung von der Norm, schreibt Stöber. Auch hier liegt ein grundsätzliches Missverständnis vor. Die Forschung beobachtet eine zunehmende Normalisierung von geschlechtergerechter Sprache (vgl. Acke 2019). Im akademischen Sprachgebrauch findet zurzeit ein Wandel der Norm statt, wie sich leicht beobachten lässt. Geschlechtergerechte Schreibweisen, welcher Art auch immer, werden zunehmend nicht nur zur ,injunktiven“ Norm (wünschenswertes Handeln), sondern auch zur „deskriptiven“ Norm (das Handeln der Mehrheit, vgl. Lapinski und Rimal 2005). Geschlechtergerechte Sprache ist damit für eine wachsende Zahl von Kolleg*innen schon längst nicht mehr ein „sprachpolitischer“ Eingriff, sondern zunehmend sprachliche Routine, der üblicherweise eine bewusste Entscheidung vorausgegangen ist.

Besonders ärgerlich an Stöbers Meinungsäußerung aber ist, dass er den Umgang mit geschlechtergerechter Sprache ohne Not zum Kulturkampf stilisiert. Er ordnet die Verwender*innen von Genderstern und Binnen-I einem ,in bestimmten Milieus verbreitete[n] illiberale[n] Klima“ zu. Offen bleibt, welche „Milieus“ damit konkret angesprochen sind und wie wir uns ein ,illiberales Klima“ vorstellen müssen. Letztlich zeigt sich aber genau hier, worin Stöbers Hauptproblem mit Genderstern und Binnen-I besteht: Er vermutet hinter deren Verwendung einen ideologischen Feldzug: „Wer bewusst sprachpolitisch eingreift, um via Sprache Denken zu lenken, manipuliert“. Dagegen lässt sich einwenden, dass die eigentliche Manipulation doch schon seit Jahrhunderten stattfindet. Wie effektiv diese Manipulation funktioniert, hat die Forschung eindrucksvoll gezeigt, zum Beispiel hinsichtlich der Wahrnehmung von Berufen als typisch ,männlich“ oder ,weiblich“ durch Kinder. Umgekehrt zeigt sich bei geschlechtergerechten Berufsbezeichnungen eine erhöhte Selbstwirksamkeit der Kinder, sodass vormals ,typisch männliche“ Berufe für alle Kinder erreichbarer erscheinen (vgl. Vervecken und Hannover 2015).

Schließlich enthält Stöbers Meinungsbeitrag einige wenig haltbare Unterstellungen. So schreibt er, dass jene, die Genderstern und Binnen-I bewusst einsetzen, ihren „Gegnern“ unterstellen, ,ideologisch ,patriarchalische“ Verhältnisse konservieren zu wollen“. Diese ziemlich unnötige ideologische Aufladung findet dann ihren vorläufigen Höhepunkt in historischen Vergleichen, bei denen man als Leser*in schnell Unwohlsein verspürt. Stöber malt nicht nur das Gespenst des Autoritarismus an die Wand, indem er George Orwells (1949) Vision von Gedankenmanipulation durch Sprachlenkung bemüht und sinister fragt: „Wo beginnt die Gedankenpolizei?“ Er bringt auch ziemlich schweres Geschütz in Stellung - für einen nicht gewinnbaren Krieg im rhetorischen Absurdistan. So möchte er jenen, die Genderstern oder Binnen-I benutzen, keinesfalls eine „Nähe zu Faschismus, Nationalsozialismus oder Kommunismus“" unterstellen. Tut das dann aber doch gleich im nächsten Satz für „manche Nutzer“. Diesen „Sprachen- und Gedankenmanipulateure[n]“ wirft Stöber vor, ,die Gesellschaft mit billiger Symbolpolitik zu indoktrinieren“ und mit „,manipulativen Volksverführern“ zu paktieren. Ein Verweis auf den „Zeitgeist“ darf 
natürlich auch nicht fehlen. All dies ,endet in der Regel mit der Aufrichtung von Guillotinen“.

Es mag sein, dass eine solche rhetorische Zuspitzung von Stöber kalkuliert war, um dem Text die nötige Aufmerksamkeit zu verschaffen. Und wenn man sich in der Fachcommunity umhört, dann ist ihm das wohl auch gelungen. Allerdings tut diese Schärfe einem konstruktiven, rationalen Diskurs der Sache (und auch dem Fach) nicht gut. Vielleicht ist es ja auch an der Zeit zu akzeptieren, dass sich an den Hochschulen dieses Landes eine Norm vor dem Hintergrund gesellschaftlich wünschenswerter Ziele verändert. Und dass die Rechtschreibung schon immer ein Werkzeug des Intellekts war, dem der Mensch zu keiner Zeit Untertan war.

Es ist an der Zeit, die veränderte Realität anzuerkennen. Die zunehmende Verwendung von Genderstern und Binnen-I (sowie ihre verwandten Formen) ist keineswegs Ausdruck einer wachsenden Missachtung der deutschen Rechtschreibung. Sie ist vielmehr Ausdruck einer sich wandelnden Sprachnorm mit dem Ziel, eine gesellschaftliche Wirklichkeit adäquat abzubilden. Etwas mehr Akzeptanz in dieser Sache würde schließlich auch der Publizistik gut zu Gesicht stehen - eine Zeitschrift, die auch in jüngerer Vergangenheit ihre Autor*innen noch dazu aufgefordert hat, die Verwendung von Genderstern und Binnen-I auf ein geringes Maß zu reduzieren.

Funding Open Access funding enabled and organized by Projekt DEAL.

Open Access Dieser Artikel wird unter der Creative Commons Namensnennung 4.0 International Lizenz veröffentlicht, welche die Nutzung, Vervielfältigung, Bearbeitung, Verbreitung und Wiedergabe in jeglichem Medium und Format erlaubt, sofern Sie den/die ursprünglichen Autor(en) und die Quelle ordnungsgemäß nennen, einen Link zur Creative Commons Lizenz beifügen und angeben, ob Änderungen vorgenommen wurden.

Die in diesem Artikel enthaltenen Bilder und sonstiges Drittmaterial unterliegen ebenfalls der genannten Creative Commons Lizenz, sofern sich aus der Abbildungslegende nichts anderes ergibt. Sofern das betreffende Material nicht unter der genannten Creative Commons Lizenz steht und die betreffende Handlung nicht nach gesetzlichen Vorschriften erlaubt ist, ist für die oben aufgeführten Weiterverwendungen des Materials die Einwilligung des jeweiligen Rechteinhabers einzuholen.

Weitere Details zur Lizenz entnehmen Sie bitte der Lizenzinformation auf http://creativecommons.org/ licenses/by/4.0/deed.de.

\section{Literatur}

Acke, H. (2019). Sprachwandel durch feministische Sprachkritik: Geschlechtergerechter Sprachgebrauch an den Berliner Universitäten. Zeitschrift für Literaturwissenschaft und Linguistik, 49(2), 303-320.

Blake, C., Klimmt, C., \& Pompetzki, V. (2008). Geschlechterrepräsentation in Nachrichtentexten. Der Einfluss von geschlechterbezogenen Sprachformen und Fallbeispielen auf den gedanklichen Einbezug von Frauen und die Bewertung der Beitragsqualität. Medien \& Kommunikationswissenschaft, 56(1), 3-20.

Hartmann, S. (2021). Gendergerecht. Forschung \& Lehre, 28(1), 40.

Irmen, L., \& Linner, U. (2005). Die Repräsentation generisch maskuliner Personenbezeichnungen. Eine theoretische Integration bisheriger Befunde. Zeitschrift für Psychologie, 213(3), 167-175.

Lapinski, M.K., \& Rimal, R. N. (2005). An explication of social norms. Communication Theory, 15(2), 127-147.

Orwell, G. (1949). Nineteen eighty-four: a novel. London: Secker \& Warburg.

Sczesny, S., \& Stahlberg, D. (2001). Effekte des generischen Maskulinums und alternativer Sprachformen auf den gedanklichen Einbezug von Frauen. Psychologische Rundschau, 52(3), 131-140. 
Sczesny, S., \& Stahlberg, D. (2005). Cognitive effects of masculine generics in German: an overview of empirical findings. Communications, 30(1), 1-21.

Vergoossen, H.P., Renström, E. A., Lindqvist, A., \& Sendén, M. G. (2020). Four dimensions of criticism against gender-fair language. Sex Roles, 83(5-6), 328-337.

Vervecken, D., \& Hannover, B. (2015). Yes I can! Effects of gender fair job descriptions on children's perceptions of job status, job difficulty, and vocational self-efficacy. Social Psychology, 46(2), 76-92.

Dr. Thomas Hanitzsch ist Professor für Kommunikationswissenschaft mit Schwerpunkt Journalismusforschung an der Ludwig-Maximilians-Universität München. 